\title{
Pressure-Induced Anomalous Phase Transitions and Colossal Enhancement of Piezoelectricity in $\mathrm{PbTiO}_{3}$
}

\author{
Zhigang Wu and Ronald E. Cohen \\ Geophysical Laboratory, Carnegie Institution of Washington, Washington, DC 20015, USA
}

(Dated: April 18, 2022)

\begin{abstract}
We find an unexpected tetragonal-to-monoclinic-to-rhombohedral-to-cubic phase transition sequence induced by pressure, and a morphotropic phase boundary in a pure compound using firstprinciples calculations. Huge dielectric and piezoelectric coupling constants occur in the transition regions, comparable to those observed in the new complex single-crystal solid-solution piezoelectrics such as $\mathrm{Pb}\left(\mathrm{Mg}_{1 / 3} \mathrm{Nb}_{2 / 3}\right) \mathrm{O}_{3}-\mathrm{PbTiO}_{3}$, which are expected to revolutionize electromechanical applications. Our results show that morphotropic phase boundaries and giant piezoelectric effects do not require intrinsic disorder, and open the possibility of studying this effect in simple systems.

PACS numbers: 77.80.-e, 77.84.-s, 63.20.-e
\end{abstract}

The classic ferroelectric $\mathrm{PbTiO}_{3}$ has been known to have a single ferroelectric tetragonal $(\mathrm{T})$ to paraelectric cubic phase transition with increased temperature [1] or pressure [2] since its discovery [3] in 1950. It seemed unlikely to discover any new ferroelectric transitions in $\mathrm{PbTiO}_{3}$, but we predict new ferroelectric phases under pressure. Piezoelectrics convert electric energy to mechanical energy, and vice versa. They are widely used in medical imaging, acoustic sensors and transducers, actuators, etc 4, 5. $\mathrm{PbTiO}_{3}$ has been extensively studied 1, 2, 6, 7, 8, 9, 10, 11 to understand the electronic origin of ferroelectricity. The $\mathrm{Pb}-\mathrm{O}$ and Ti-O hybridization in $\mathrm{PbTiO}_{3}$ weakens the short-range repulsions and gives rise to the ferroelectric distortion 7]. It has been found that pressure suppresses ferroelectricity since compression favours short-range repulsion, but there has been no previous work on the effect of pressure on piezoelectricity.

$\mathrm{PbTiO}_{3}$ has a high $c$-axis strain of $6.5 \%(7.1 \%)$ at room (low) temperature. Under ambient pressure it undergoes a first-order phase transition at $T_{\mathrm{c}}=763 \mathrm{~K}$, and this transition is regarded as a typical displacive transition, which is associated with soft-modes [1]. The displacive phase transition temperature $T_{\mathrm{c}}$ decreases under hydrostatic pressure. A Raman study of $\mathrm{PbTiO}_{3}$ shows that $T_{\mathrm{c}}$ reduces to $300 \mathrm{~K}$ and the phase transition becomes second-order at $P=12.1 \mathrm{GPa} 2$. It indicates a tricritical point in the phase diagram, where the first-order phase transition changes to second-order, and it has been identified at $P=1.75 \mathrm{GPa}, T=649 \mathrm{~K} 12$. Increasing pressure further reduces $T_{\mathrm{c}}$.

$\mathrm{PbTiO}_{3}$ is an end member of $\mathrm{Pb}\left(\mathrm{Mg}_{1 / 3} \mathrm{Nb}_{2 / 3}\right) \mathrm{O}_{3}-$ $\mathrm{PbTiO}_{3}$ (PMN-PT) and $\mathrm{Pb}\left(\mathrm{Zn}_{1 / 3} \mathrm{Nb}_{2 / 3}\right) \mathrm{O}_{3}-\mathrm{PbTiO}_{3}$ (PZN-PT), which have piezoelectric coefficients an order of magnitude larger than those of conventional ferroelectric simple compounds [13]. It is also an end member of $\mathrm{PbZrO}_{3}-\mathrm{PbTiO}_{3}(\mathrm{PZT})$, the most widely used current piezoelectric, which is ubiquitous in modern technology. These materials have a common feature, namely the morphotropic phase boundary (MPB), and they have optimum piezoelectric efficiency near the MPB. Polar- ization rotation [14, 15] is believed to play an important role for this extraordinary property. Close to the MPB, the energy surface for polarization is very flat so that for example a polarization along [111] direction in the rhombohedral $(\mathrm{R})$ phase can be easily rotated toward the tetragonal polarization direction by applying an electric field along [001] direction, and the $\mathrm{R}$ phase is transformed to the $\mathrm{T}$ phase via the intermediate phase(s). This mechanism has been elucidated by theoretical calculations on $\mathrm{BaTiO}_{3}$ [14] and PZT [16, 17], and by experimental findings of low symmetry phases 18, 19, 20]. The giant piezoelectric effects always do not occur along the spontaneous polarization direction [13, 15]). At ambient pressure $\mathrm{PbTiO}_{3}$ has no such MPB because it has a rather stiff energy surface near the $T$ phase and it has no $R$ phase. Under high pressures the energy (enthalpy) surface is expected to be softer, and an MPB could arise.

In this Letter we address both issues of phase transitions and piezoelectricity of $\mathrm{PbTiO}_{3}$ under hydrostatic pressures. We perform total energy as well as linear response computations, which have been proved to be highly reliable for ground state properties. We find anomalous phase transitions and giant enhancement of dielectricity and piezoelectricity near the phase transition regions induced by pressure.

We have carried out zero temperature ab initio computations based on density functional theory (DFT) within the local density approximation (LDA). We used the pseudopotential planewave method implemented in the ABINIT package 21. The planewave energy cutoff is 60 Hartree, and the k-point mesh for Brillouin Zone integration is of $6 \times 6 \times 6$. We used the OPIUM program 22 to generate norm-conserving pseudopotentials, which were rigorously tested against the full-potential linearized augmented planewave (LAPW) method 23. We included semi-core states of $\mathrm{Pb} 5 d^{10}$, Ti $3 s^{2} 3 p^{6} 4 d^{2}$, and $\mathrm{O} 2 s^{2}$ in valence states. We chose the LDA instead of the generalized gradient approximation (GGA) because the GGA catastrophically overestimates both equilibrium volume and strain for tetragonal $\mathrm{PbTiO}_{3}$ 24]. On the other 
TABLE I: Elastic $\left(c_{\mu \nu}\right)$ and piezoelectric $\left(e_{i \nu}\right)$ constants of tetragonal $\mathrm{P}_{4 m} \mathrm{~m} \mathrm{PbTiO}_{3}$ with lattice constants $a=3.902 \AA$, $c=4.155 \AA$. $c_{\mu \nu}$ and $e_{i \nu}$ are in $\mathrm{GPa}$ and $\mathrm{C} / \mathrm{m}^{2}$, respectively. Here FS refers to the finite strain method.

\begin{tabular}{l|ccccccccc}
\hline \hline Methods & $c_{11}$ & $c_{12}$ & $c_{13}$ & $c_{33}$ & $c_{44}$ & $c_{66}$ & $e_{31}$ & $e_{33}$ & $e_{15}$ \\
\hline DFPT & 230 & 96.2 & 65.2 & 41.9 & 46.6 & 98.8 & 2.06 & 4.41 & 6.63 \\
FS & 229 & 95.6 & 64.3 & 41.2 & 47.2 & 98.6 & 2.07 & 4.48 & 6.66 \\
\hline \hline
\end{tabular}

hand, the LDA moderately underestimates the volume $\left(60.38 \AA^{3}\right)$ and strain $(4.6 \%)$, and the experimental $V_{0}$ corresponds to a negative pressure $P_{0}=-2.2 \mathrm{GPa}$.

To calculate the dielectric susceptibility $\chi_{i j}$, elastic constants $c_{\mu \nu}$, and piezoelectric stress constants $e_{i \nu}$ (Here Latin indexes run from 1 to 3 , and Greek indexes from 1 to 6 ), we used the density functional perturbation theory (DFPT) 25 of the linear response of strain type perturbations [26]. The DFPT is based on the systematic expansion of the variation expression of the DFT total energy in powers of parameters, such as atomic coordinates, macroscopic strain and electric field. Tensors $\chi$ and $c$ involve second derivatives of total energy with respect to electric field and strain respectively, and tensor $e$ is the mixed second derivative of total energy with respect to strain and electric field. Here $c_{\mu \nu} \equiv \frac{\partial \sigma_{\mu}}{\partial \eta_{\nu}}$ with $\sigma$ stress and $\eta$ strain. The DFPT calculates $C_{\mu \nu}=\frac{\partial^{2} E}{\partial \eta_{\mu} \partial \eta_{\nu}}$ with $E$ the total energy. Under non-zero stress (e.g. pressure), $c_{i j k l}=C_{i j k l}-\delta_{i j} \sigma_{k l}$. The elastic compliance tensor $s$ is the reciprocal of tensor $c$, and the piezoelectric strain coefficients $d_{i \nu}=\sum_{\mu=1}^{6} e_{i \mu} s_{\mu \nu}$. We have examined the accuracy of $c_{\mu \nu}$ and $e_{i \nu}$ obtained from the DFPT by comparing with the finite strain method. As summarized in Table [ excellent agreement between these two methods is achieved.

We constrained the symmetry to study four phases: the paraelectric cubic (C) $P m \overline{3} m$ phase, the ferroelectric tetragonal $P 4 m m$, rhombohedral $R 3 m$, and monoclinic (M) $\mathrm{Cm}$ phases. We find that the enthalpy $(H=E+P V)$ difference between the $\mathrm{T}$ and $\mathrm{R}$ phases reduces rapidly with pressure and becomes very small when $P>8 \mathrm{GPa}$. The lowest enthalpy corresponds to the most stable phase at that pressure. As displayed in Fig. 1(a) and $1(\mathrm{~b})$, there is a $\mathrm{T}$ to $\mathrm{M}$ phase transition around 9.5 $\mathrm{GPa}\left(V=56.73 \AA^{3}\right), \mathrm{M}$ to $\mathrm{R}$ around $11 \mathrm{GPa}(V=56.32$ $\left.\AA^{3}\right)$, and $\mathrm{R}$ to $\mathrm{C}$ around $22 \mathrm{GPa}\left(V=53.97 \AA^{3}\right)$. The M phase for $P=9.5 \mathrm{GPa}$ has its spontaneous polarization $\mathbf{P}^{\mathrm{s}}$ along the pseudocubic [uu1] direction, where $u=0.481$. The discovery of a low symmetry M phase is completely unexpected in a pure compound like $\mathrm{PbTiO}_{3}$, and has only been found in complex solid-solutions like PZT [18], PMN-PT [19], etc.

Because the enthalpy differences among these phases are very small, the above calculated pressure-induced phase transition sequence needs to be examined by directly studying energy derivative properties, such as elas-
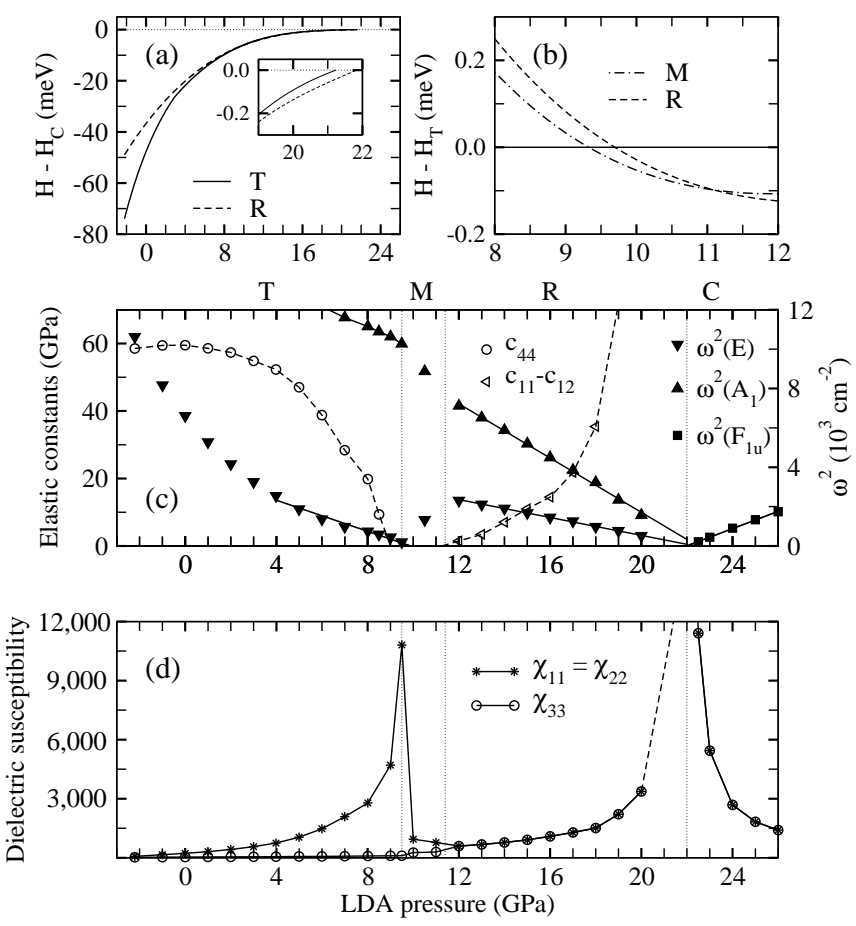

FIG. 1: Stability of various phases as a function of pressure. (a) Enthalpy difference with respect to the $\mathrm{C}$ phase for the $\mathrm{T}$ and $\mathrm{R}$ phases. (b) Enthalpy difference with respect to the $\mathrm{T}$ phase for the $\mathrm{R}$ and $\mathrm{M}$ phases. (c) Elastic constant $c_{44}$ and $c_{11}-c_{12}$ respectively for the $\mathrm{T}$ and $\mathrm{R}$ phases, and square of the lowest optical phonon frequencies $\omega^{2}$ for the $\mathrm{T}[E(1 \mathrm{TO})$, $\left.A_{1}(1 \mathrm{TO})\right], \mathrm{R}\left[E(1 \mathrm{TO}), A_{1}(1 \mathrm{TO})\right]$, and $\mathrm{C}\left[F_{1 u}(1 \mathrm{TO})\right]$ phases. (d) Dielectric susceptibility $\chi$.

ticity, phonon frequencies, and dielectricity. The pressure dependence of elastic constants $c_{44}$ in the $\mathrm{T}$ phase and $c_{11}-c_{12}$ in the $\mathrm{R}$ phase is shown in Fig. 1(c). Note that the cubic coordinate system is used for all cases, and elastic constants $c_{11}=c_{22}=c_{33}$ and $c_{12}=c_{13}=c_{23}$ in the $\mathrm{R}$ phase. We find that both $c_{44}$ and $c_{11}-c_{12}$ approach zero around 9 and $11.5 \mathrm{GPa}$, respectively. Negative $c_{44}$ and $c_{11}-c_{12}$ mean that the $\mathrm{T}$ and $\mathrm{R}$ phases are unstable respectively against shear and tetragonal shear strain, indicating phase transitions. The estimated phase transition pressures from elasticity anomalies agree well with the total energy results.

In tetragonal $P 4 m m \quad \mathrm{PbTiO}_{3}$, the $E(1 \mathrm{TO})$ and $A_{1}(1 \mathrm{TO})$ modes originate from the triply degenerate $F_{1 u}(1 \mathrm{TO})$ mode in the $\mathrm{C}$ phase. When the T-to-C phase transition occurs at high temperature and ambient pressure [1] or high pressure and room temperature 2], both the $E(1 \mathrm{TO})$ and $A_{1}(1 \mathrm{TO})$ modes soften simultaneously. However, at $0 \mathrm{~K}$ we find that pressure induces condensation only of the $E(1 \mathrm{TO})$ mode around $P=10 \mathrm{GPa}$ [Fig. 1(c)]. A linear combination of the doubly degenerate $E(1 \mathrm{TO})$ modes gives rise to the transition to the $\mathrm{M}$ phase. For the $\mathrm{R}$ phase, the $E(1 \mathrm{TO})$ and $A_{1}(1 \mathrm{TO})$ 


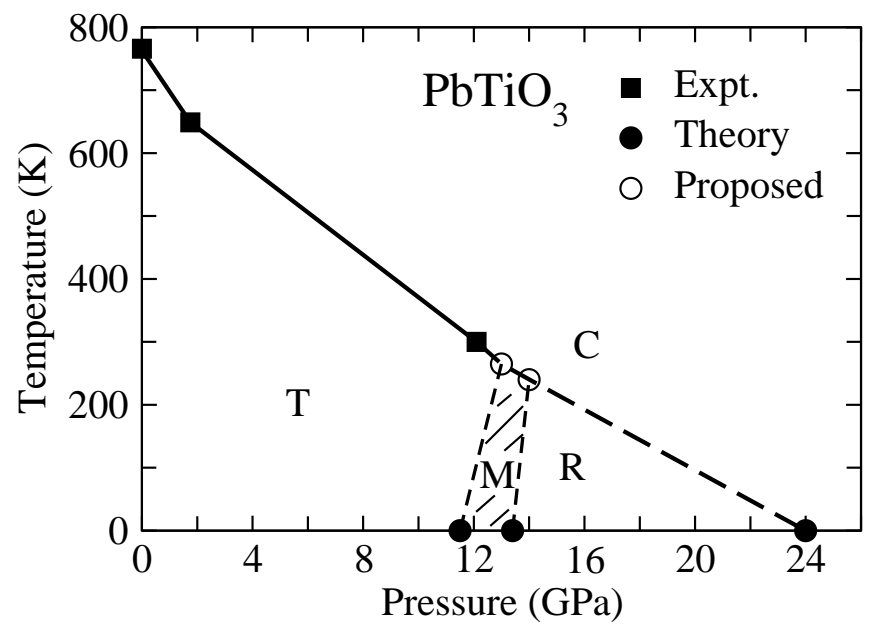

FIG. 2: Proposed $(P, T)$ phase diagram. It combines theoretical results (solid circles) at $0 \mathrm{~K}$ with experimental data (solid squares) at finite temperatures. In this figure we rescaled the theoretical pressure for the experimental volume to be zero by shifting. The open circles are our guess for these two tricritical points, and an ab initio molecular dynamics simulation is needed to predict them accurately.

modes soften simultaneously at $P \approx 22 \mathrm{GPa}$, going to the $F_{1 u}(1 \mathrm{TO})$ mode in the $\mathrm{C}$ phase. This gives rise to the R-to-C phase transition. The linearity of the square of mode frequency $\omega^{2}$ vs. pressure close to phase transition indicates a Curie-Weiss pressure law. Fig. 1(d) shows that the static dielectric susceptibility $\chi_{11}$ has a maximum around 9.5 GPa, indicating a first-order transition; while it diverges at $P \approx 22 \mathrm{GPa}$, indicating a second-order transition, as can be understood from the Lyddane-Sachs-Teller (LST) relation. For the T phase near transition pressure, $\chi_{11}=\chi_{22} \propto 1 / \omega^{2}[E(1 T O)]$, and $\chi_{33} \propto 1 / \omega^{2}\left[A_{1}(1 T O)\right]$. Thus $\chi_{11}$ increases rapidly around $9.5 \mathrm{GPa}$, whereas $\chi_{33}$ does not. The same analysis can be applied to the $\mathrm{R}$ and $\mathrm{C}$ phases. The phase transition pressures obtained from phonon frequencies and dielectric constants also are consistent with the total energy results, and it shows that the computed small energy differences between phases are reliable. Our first-principles calcalations neglect quantum fluctuations, which could reduce the phase transition pressures 27.

We have predicted the pressure-induced phase transitions of $\mathrm{PbTiO}_{3}$ at $0 \mathrm{~K}$. Combining our results with experimental finite temperature data, we propose a schematic $(P, T)$ phase diagram (Fig. 2), in which the intermediate $\mathrm{M}$ phase separates the $\mathrm{T}$ phase at low pressures and the $\mathrm{R}$ phase at higher pressures. This phase diagram bears remarkable resemblance to the $(x, T)$ phase diagram of PZT [18], where $x$ is the $\mathrm{PbTiO}_{3}$ composition. The narrow $\mathrm{M}$ area is the $\mathrm{MPB}$ of $\mathrm{PbTiO}_{3}$, and it serves as a structure bridging the $\mathrm{T}$ and $\mathrm{R}$ phases in that its spontaneous polarization is located in the (110) plane
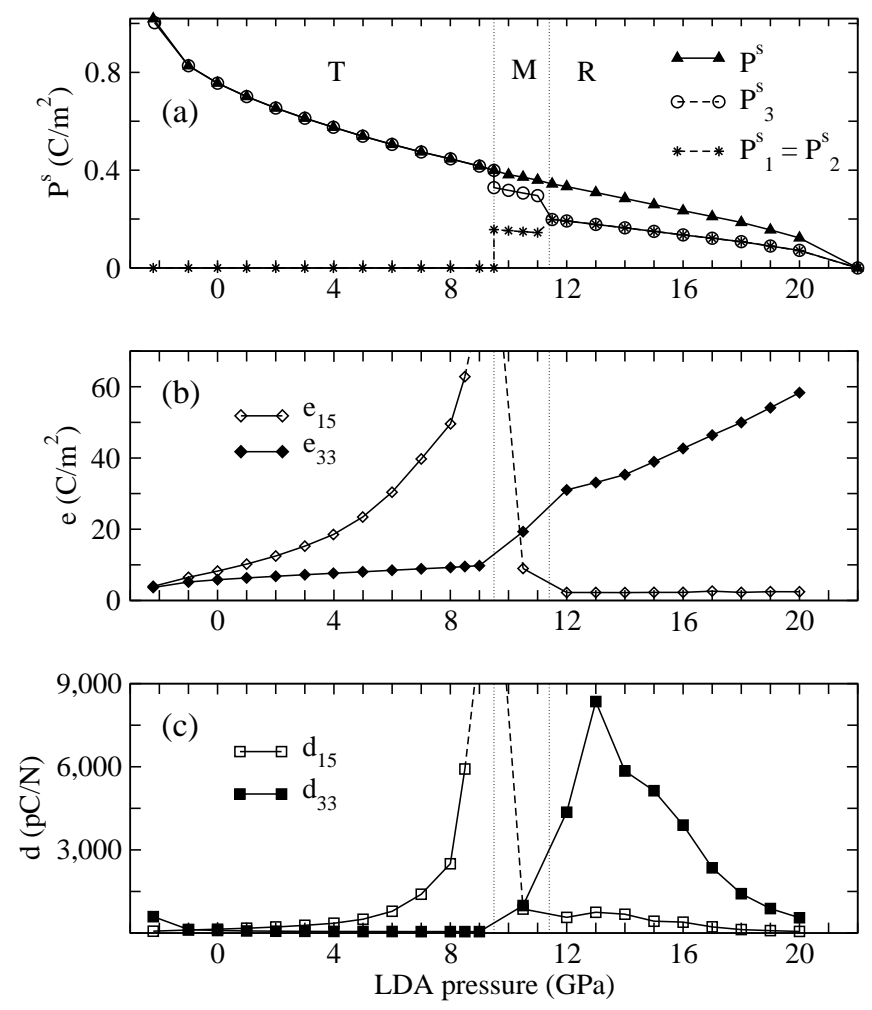

FIG. 3: Pressure dependence on piezoelectricity. (a) Spontaneous polarization $\mathbf{P}^{\mathrm{s}}$. (b) Piezoelectric stress coefficients $e_{15}$ and $e_{33}$. (c) Piezoelectric strain coefficients $d_{15}$ and $d_{33}$.

and between the pseudocubic [001] and [111] directions, which is very similar to that in PZT.

Pressure suppresses spontaneous polarization $\mathbf{P}^{\mathrm{s}}$ [Fig. $3(\mathrm{a})]$ and the $c$-axis strain. Although the magnitude of $\mathbf{P}^{\mathrm{s}}$ changes continuously at the T-M transition pressure, $P_{1}^{\mathrm{s}}$ is discontinuous, indicating a first-order phase transition, consistent with the conclusion drawn from dielectric constants. We emphasize that when the T-to-M phase transition occurs, $\mathbf{P}^{\mathrm{s}}$ retains about half of the magnitude under zero pressure and $c / a=1.012$. These values are comparable to those of PMN-PT [19], PZN-PT [20], and PZT 28] in the T phase close to MPB.

Fig. 3(b) and 3(c) summarize the pressure effect on piezoelectricity of $\mathrm{PbTiO}_{3}$. For the $\mathrm{T}$ phase, $e_{33}$ increases, whereas the piezoelectric strain coefficient $d_{33}$ decreases with pressure. The relatively large $d_{33}$ at $P=-2.2 \mathrm{GPa}$ is due to the LDA overestimation of strain at the experimental volume. Both $e_{15}$ and $d_{15}$ increase with pressure, and they rise dramatically for $P>4 \mathrm{GPa}$ when $c_{44}$ begins to drop quickly [Fig. 1(c)]. The T phase has $d_{15}=e_{15} / c_{44}$, so that $d_{15}$ increases even faster than $e_{15}$. The pressure-induced large enhancements of $e_{15}$ and $d_{15}$ are a result of the sharp reduction of the enthalpy difference between the $\mathrm{T}$ and $\mathrm{R}$ phases, and both $e_{15}$ and $d_{15}$ diverge at the T-to-M phase transition pressure. For 
the R phase, both $e_{15}$ and $d_{15}$ are rather small, and $e_{33}$ jumps to over $30 \mathrm{C} / \mathrm{m}^{2}$, increasing with pressure. $d_{33}$ has huge values near the $\mathrm{M}$ phase and remains larger than $1500 \mathrm{pN} / \mathrm{C}$ for a broad range of pressure. The $\mathrm{R}$ phase under high pressure has $d_{33} \approx e_{33} /\left(c_{11}-c_{12}\right)$, and the increase of $c_{11}-c_{12}$ with pressure [Fig. 1(c)] causes $d_{33}$ to decrease with pressure, even though $e_{33}$ rises. Note that $d_{33}$ at $12 \mathrm{GPa}$ is smaller that at $13 \mathrm{GPa}$, and it could be due to numerical uncertainties, since small variations of elastic constants $c_{14}$ and $c_{15}$ change elastic compliance $s$ dramatically when $c_{11}-c_{12}$ is small. It is evident that the predicted giant piezoelectric effect of $\mathrm{PbTiO}_{3}$ comes from non-collinear polarization rotation since the enhancement of $d_{15}$ in the $\mathrm{T}$ phase and $d_{33}$ in the $\mathrm{R}$ phase do not occur along the spontaneous polarization directions, and the $\mathrm{M}$ phase acts as a structural bridge between the $\mathrm{T}$ and $\mathrm{R}$ phases as indicated by pressure inducing polarization rotation from [001] to [111] directions.

We have demonstrated that pressure can induce an MPB in a simple compound $\mathrm{PbTiO}_{3}$, which is very similar to the composition-induced MPB in complex solidsolutions, such as PZT [18], PMN-PT [19], and PZN-PT [20]. It is critical that these ferroelectric systems near MPB have distinct phases (different patterns of atomic displacements) with very close free energies. Each local minimum is shallow and a broad global minimum exists. Low symmetry phases can be stabilized between two high symmetry phases if both of them become saddle points, and this is the origin of an MPB. Near an MPB, smooth transformation between two high symmetry phases with different polarization directions via a low symmetry phase results in strong coupling between internal degree of freedom and strain.

In $\mathrm{PbTiO}_{3}$, ferroelectricity arises from the competition of short-range repulsions which favor the $\mathrm{C}$ phase, and Coulomb forces which favor a ferroelectric phase [7]. As pressure is increased, the short-range repulsions promoting paraelectric stability increase faster than the Coulomb terms promoting ferroelectric instability. Therefore pressure favors the paraelectric phase and suppresses ferroelectricity. Because the tetragonal welldepth along [001] reduces faster than the rhombohedral wells along [111], under a certain pressure the $\mathrm{T}$ and $\mathrm{R}$ well-depths are equal, and the energy surface connecting the $\mathrm{T}$ and $\mathrm{R}$ phase becomes very flat. Higher order terms in the energy expression result in an intermediate M phase [29], which has slightly lower energy than both of the $\mathrm{T}$ and $\mathrm{R}$ phases, leading to a T-to-M phase transition. Further increasing pressure will make the $\mathrm{R}$ phase more stable than the $\mathrm{M}$ phase. For another important ferroelectric, $\mathrm{BaTiO}_{3}$, an $\mathrm{M}$ phase is not expected at low temperature, because the $\mathrm{R}$ well-depth is deeper than the $\mathrm{T}$ well-depth so that pressure can not induce an $\mathrm{M}$ phase before both well-depths disappear.

Pressure-tuning of the structural and piezoelectric properties of $\mathrm{PbTiO}_{3}$ promises to be an intriguing avenue for experimental study. $\mathrm{PbTiO}_{3}$ under pressure should be the simplest possible system to study the basic physics of the MPB and piezoelectric enhancement through polarization rotation. The predicted monoclinic $\mathrm{Cm}$ phase (without strain and external electric field) and the MPB accompanied by giant piezoelectric effects in simple perovskite compounds have never yet been reported. Very recently, Rouquette et al. reported experimental evidence of polarization rotation induced by pressure in PZT [30, 31, 32. We hope our predictions will stimulate both fundamental and technological interests to investigate ferroelectrics under hydrostatic or uniaxial pressure, and stress and pressure as means to enhance piezoelectricity.

We thank fruitful discussions with N. Choudhury and E. J. Walter. Computations were done on the Center for Piezoelectrics by Design. This work was supported by the Office of Naval Research under ONR Grants No. N00014-02-1-0506.

[1] G. Burns and B. A. Scott, Phys. Rev. Lett. 25, 167 (1973).

[2] J. A. Sanjurjo, E. López-Cruz, and G. Burns, Phys. Rev. B 28, 7260 (1983).

[3] G. Shirane, S. Hoshino, and K. Suzuki, Phys. Rev. 80, 1105 (1950).

[4] M. E. Lines and A. M. Glass, Principles and Applications of Ferroelectrics and Related Materials (Clarendon Press, Oxford, 1977).

[5] K. Uchino, Piezoelectric acutuators and ultrasonic motors (Kluwer Academic, Boston, 1996).

[6] Chemical Abstracts reports 9924 publications on $\mathrm{PbTiO}_{3}$ since its discovery.

[7] R. E. Cohen, Nature (London) 358, 136 (1992).

[8] A. García and D. Vanderbilt, Phys. Rev. B 54, 3817 (1996).

[9] U. V. Waghmare and K. M. Rabe, Phys. Rev. B 55, 6161 (1997).

[10] G. Sághi-Szabó, R. E. Cohen, and H. Krakauer, Phys. Rev. Lett. 80, 4321 (1998).

[11] Z. Wu, G. Sághi-Szabó, R. E. Cohen, and H. Krakauer, Phys. Rev. Lett. 94, 069901 (2005).

[12] R. Ramirez, M. F. Lapena, and J. A. Gonzalo, Phys. Rev. B 42, 2604 (1990).

[13] S. E. Park and T. R. Shrout, J. of Appl. Phys. 82, 1804 (1997).

[14] H. Fu and R. E. Cohen, Nature (London) 403, 281 (2000).

[15] B. Noheda, et al., Phys. Rev. Lett. 86, 3891 (2001).

[16] L. Bellaiche, A. García, and D. Vanderbilt, Phys. Rev. Lett. 84, 5427 (2000).

[17] Z. Wu and H. Krakauer, Phys. Rev. B 68, 014112 (2003).

[18] B. Noheda, et al., Appl. Phys. Lett. 74, 2059 (1999).

[19] B. Noheda, D. E. Cox, G. Shirane, J. Gao, and Z.-G. Ye, Phys. Rev. B 66, 054104 (2002).

[20] D. La-Orauttapong, et al., Phys. Rev. B 65, 144101 
(2002).

[21] X. Gonze, et al., Comput. Mater. Sci. 25, 478 (2002).

[22] A. M. Rappe, K. M. Rabe, E. Kaxiras, and J. D. Joannopoulos, Phys. Rev. B 41, 1227 (1990).

[23] D. J. Singh, Planewaves, Pseudopotentials, and the LAPW Method (Kluwer Academic Publishers, Boston, 1994).

[24] Z. Wu, R. E. Cohen, and D. J. Singh, Phys. Rev. B 70, 104112 (2004).

[25] S. Baroni, S. de Gironcoli, A. Dal Corso, and P. Giannozzi, Rev. Mod. Phys. 73, 515 (2001).
[26] D. R. Hamann, X. Wu, K. M. Rabe, and D. Vanderbilt, Phys. Rev. B 71, 035117 (2005).

[27] J. ÍñiguezD. Vanderbilt, Phys. Rev. Lett. 89, 115503 (2002).

[28] B. Noheda, et al., Phys. Rev. B 63, 014103 (2001).

[29] D. Vanderbilt and M. H. Cohen, Phys. Rev. B 63, 094108 (2001).

[30] J. Rouquette, et al., Phys. Rev. B 71, 024112 (2005).

[31] J. Rouquette, et al., Phys. Rev. B 70, 014108 (2004).

[32] J. Rouquette, et al., Phys. Rev B 65, 214102 (2002)) 